\title{
ATOMIC PARITY NONCONSERVATION: ELECTROWEAK PARAMETERS AND NUCLEAR STRUCTURE
}

\author{
S.J. Pollock \\ Institute for Nuclear Theory, University of Washington, Seattle, WA 98195 \\ E. N. Fortson and L. Wilets \\ Department of Physics, University of Washington, Seattle, WA 98195
}

(Received May 12 1992)

There have been suggestions to measure atomic parity nonconservation (PNC) along an isotopic chain, by taking ratios of observables in order to cancel complicated atomic structure effects. Precise atomic PNC measurements could make a significant contribution to tests of the Standard Model at the level of one loop radiative corrections. However, the results also depend upon certain features of nuclear structure, such as the spatial distribution of neutrons in the nucleus. To examine the sensitivity to nuclear structure, we consider the case of $\mathrm{Pb}$ isotopes using various recent relativistic and non-relativistic nuclear model calculations. Contributions from nucleon internal weak structure are included, but found to be fairly negligible. The spread among present models in predicted sizes of nuclear structure effects may preclude using $\mathrm{Pb}$ isotope ratios to test the Standard Model at better than a one percent level, unless there are adequate independent tests of the nuclear models by various alternative strong and electroweak nuclear probes. On the other hand, sufficiently accurate atomic PNC experiments would provide a unique method to measure neutron distributions in heavy nuclei. 
12.15.Ji,12.15.Mm,21.60.-n,31.10+z

Typeset Using REVTEX 


\section{INTRODUCTION}

Parity nonconservation (PNC) in atoms arises from the electroweak interaction between the electrons and nucleons, primarily due to exchange of the neutral gauge boson, $Z_{0}$. The dominant contribution in heavy atoms comes from the coupling of the axial electronic current to the vector nucleon current. Because the vector currents are conserved, atomic PNC essentially measures the electroweak coupling to the elementary quarks, bypassing many of the difficulties of hadronic physics. Thus in principle atomic experiments can measure certain key electroweak parameters quite accurately, and also help probe for new physics beyond the presently successful Standard Model of the electroweak interactions.

In fact, there remains much to be learned about the Standard Model, including the masses of the top quark and the predicted Higgs boson(s), and whether there are additional generations of quarks and leptons. In addition the Standard Model faces the well-known gauge hierarchy problem, and it is certainly possible that electroweak measurements may reveal something totally new, such as technicolor or supersymmetric particles. Accurate measurements of PNC in atomic cesium already play an important role in addressing such questions.

Two major issues affect the interpretation of atomic experiments and will become more crucial as experimental accuracy improves, namely the small but not negligible effects of nuclear size and structure, [1] and the reliability of the atomic theory of heavy atoms [2,3]. Atomic theory, the source of the largest uncertainty, has received a great deal of attention leading to increasingly precise calculations of PNC for a number of elements. Cesium, in particular, is now believed to be understood at the $1 \%$ level.

To advance further may require canceling out all uncertainties of the atomic theory by comparing PNC measurements on different isotopes of the same element. Such experiments in fact have been proposed [4 using strings of isotopes of such elements as $\mathrm{Cs}, \mathrm{Dy}$, and $\mathrm{Pb}$.

As we discussed in a previous note, [1] hereafter referred to as I, it then becomes important to find the level at which nuclear structure interferes with interpreting atomic PNC 
purely in terms of particle theory. The wave function of the atomic electrons varies over the dimensions of the nucleus, causing the net electroweak interaction with the nucleons to depend on the spatial distribution of both the protons and neutrons. As demonstrated in I, the PNC observable is (for $\sin ^{2} \theta_{W} \approx 1 / 4$ and $R_{n} \approx R_{p}$ ) very roughly proportional to

$$
1-\frac{3}{70}(Z \alpha)^{2}\left[1+5 R_{n}^{2} / R_{p}^{2}\right]+\cdots
$$

where $R_{n}$ and $R_{p}$ are the equivalent rms radii for the nuclear distribution of neutrons and protons. The proton, or rather the nuclear charge, distribution is well known from electric probes: electron and muon scattering, optical isotope shifts, muonic atoms, etc. The extraction of the neutron distribution, however, is quite model-dependent and difficult to determine to the same high accuracy.

On the one hand, the neutron distribution is needed in order to extract the weak parameters in heavy atom experiments. On the other hand, to the extent the weak parameters are known, the experiments provide a method of measuring the changes in the neutron distribution, primarily the rms radius. Thus atomic experiments on isotopes of heavy atoms may provide a unique opportunity to test nuclear model calculations.

In this paper we explore the nuclear structure issues extensively. We have utilized several recent detailed nuclear structure calculations from various authors, in order to quantitatively estimate the nuclear model-dependent corrections to atomic PNC in the isotopes of ${ }_{82} \mathrm{~Pb}$, an element of interest experimentally. Our major conclusions are:

(1) For single isotope measurements on ${ }^{208} \mathrm{~Pb},(Z \alpha)$ is sufficiently large that the effects of nuclear structure on atomic PNC cannot be neglected. The uncertainties due to neutron distributions appear to be less significant for the extraction of electroweak parameters than the current uncertainties due to atomic structure. However, unless the change in neutron distributions along an isotopic chain can be better predicted (or independently measured, e.g. via parity violating $\mathrm{e}^{-}$scattering), $\mathrm{PNC}$ ratios of ${ }_{82} \mathrm{~Pb}$ isotopes will not be able to provide an extraction of the weak mixing angle to better than a one percent level.

(2) In lighter nuclei (including the important case of ${ }_{55} \mathrm{Cs}$ ), $Z \alpha$ is sufficiently small 
that uncertainties in nuclear structure can probably be safely ignored compared to current uncertainties in atomic theory, when using a single isotope. Calculations for the non-magic, odd- $Z$, Cs nuclei pose additional difficulties, and further investigation is still required to determine how accurately the Standard Model could be tested when using ratios of isotopes.

(3) Atomic PNC experiments provide perhaps one of the cleanest opportunities yet available to study the nuclear neutron skin. The situation is similar to atomic isotope shifts which have provided precise measurements of the ratios of changes in the nuclear rms charge radius in strings of isotopes. Here we have a weak probe of the neutron distribution which is free of the gross uncertainties associated with strongly interacting probes. At the level of precision that the Standard Model is known, this yields another testing ground for nuclear models.

We note that alternative weak probes, such as parity-violating intermediate-energy $e^{-}$nucleus cross section asymmetries, are also sensitive to the neutron distributions. In combination with atomic PNC, these may help simplify the separation of nuclear structure effects from electroweak radiative corrections.

The paper is organized as follows: In section II we sketch briefly the relevant parts of electroweak theory. In section III we review the simple analytical model we presented in reference (1); this provides a convenient framework for discussing the effects of nuclear structure on atomic PNC in terms of the rms radii of the proton and neutron distributions in the nucleus. In section IV we discuss the electroweak nucleon form factors. The intrinsic electroweak structure does begin to contribute at the level we are interested in, but the uncertainties in this structure (due to strangeness admixtures, etc.) should have a negligible effect on the total PNC amplitude of the nucleus. In section $\mathrm{V}$, we examine the key ingredients of currently available theoretical models for heavy nuclei, including both non-relativistic and relativistic formalisms. We consider the reliability of these models, and discuss the need for calculations which include correlations beyond the Hartree-Fock level. We also discuss alternative experimental means to measure the desired neutron distributions. In section VI we discuss the relevant Standard Model parameters, and the accuracy desired in their extraction from atomic parity violation. We derive the propagation of error 
from nuclear model uncertainties to electroweak parameters, focusing on isotopes of lead and (to a lesser extent) cesium. These two elements are of current experimental interest, and are representative of the very heavy and moderately heavy regions of the periodic table. In section VII we discuss our numerical results, using various existing Hartree-Fock nuclear calculations, summarize, and discuss the conclusions from the previous sections.

\section{PNC IN THE STANDARD MODEL}

\section{A. Theoretical Considerations}

Because the $Z$-boson is massive $(91.16 \pm .03 \mathrm{GeV})$, the quark-electron interaction due to $Z$-exchange may be taken to be of zero range compared to atomic or nuclear dimensions. What we observe in atoms is the electron interaction with nucleons, not individual quarks. Nucleons are, of course, composite structures consisting each of three quarks net, but also $\bar{q} q$

pairs as well as gluons. We make the assumption here, which we justify in Sec. IV, that we can neglect the internal nucleon structure and simply add the point coupling of the 3 quarks to obtain the net nucleon weak coupling. The PNC part of the nucleon-electron interaction can be written in terms of axial and vector currents

$$
H_{P N C}=V_{N} \times A_{e}+A_{N} \times V_{e} .
$$

If, in addition to neglecting internal nucleon structure, we treat the nucleons nonrelativistically (a very good approximation), we have

$$
\begin{aligned}
H_{P N C}=\frac{G_{F}}{\sqrt{2}} \sum_{e B}[ & C_{1 B} \int \psi_{B}^{\dagger} \psi_{B} \psi_{e}^{\dagger} \gamma^{5} \psi_{e} d^{3} r \\
& \left.+C_{2 B} \int \psi_{B}^{\dagger} \vec{\sigma}_{B} \psi_{B} \cdot \psi_{e}^{\dagger} \vec{\alpha} \psi_{e} d^{3} r\right],
\end{aligned}
$$

where $B$ stands for $n$ (neutron) or $p$ (proton) and

$$
C_{1 p}=\frac{1}{2}\left(1-4 \sin ^{2} \theta_{W}\right)
$$




$$
\begin{aligned}
& C_{2 p}=\frac{1}{2}\left(1-4 \sin ^{2} \theta_{W}\right) g_{A}, \\
& C_{1 n}=-\frac{1}{2}, \\
& C_{2 n}=-\frac{1}{2}\left(1-4 \sin ^{2} \theta_{W}\right) g_{A} .
\end{aligned}
$$

These expressions assume tree-level Standard Model couplings. In section VI, we discuss the important effects of radiative loop corrections.

The first term in eqn. (2.2a) grows coherently with nucleon numbers $N$ and $Z$. The second term, together with the anapole moment [5] term (which also depends upon $\vec{\sigma}_{B} \cdot \vec{\alpha}$ ), amounts to at most a few percent of the first term in heavy atoms, and furthermore sums to zero when all hfs sublevels are combined, since all directions of $\vec{\sigma}_{B}$ are then weighted equally. Thus in this paper we will consider the first term only. The effective interaction is

$$
\begin{aligned}
H_{P N C, 1}=\frac{G_{F}}{2 \sqrt{2}} \int[ & \left.-N \rho_{n}(\vec{r})+Z\left(1-4 \sin ^{2} \theta_{W}\right) \rho_{p}(\vec{r})\right] \\
& \times \psi_{e}^{\dagger} \gamma^{5} \psi_{e} d^{3} r
\end{aligned}
$$

where here the $\rho_{n}$ and $\rho_{p}$ are normalized to unity. The neutron and proton densities include a folding with the weak form factors (see Sec. IV).

We need the spatial variation of $\psi_{e}^{\dagger} \gamma^{5} \psi_{e}$ over the nucleus, its normalization, and its dependence on nuclear structure. PNC effects are dominated by $s_{1 / 2}$-electrons $(\kappa=-1)$ coupled to $p_{1 / 2}$-electrons $(\kappa=+1)$. We define

$$
\rho_{5}(r) \equiv \psi_{p}^{\dagger}(\vec{r}) \gamma^{5} \psi_{s}(\vec{r})
$$

which turns out to depend only on the magnitude of $\vec{r} . \rho_{5}(r)$ can be factored conveniently as follows:

$$
\rho_{5}(r)=C(Z) \mathcal{N}(Z, R) f(r),
$$

where $C(Z)$ contains all atomic structure effects for a point nucleus including many-body correlations; $\mathcal{N} \equiv \psi_{p}^{\dagger}(0) \gamma^{5} \psi_{s}(0)$ is the normalization factor for a single electron; $f(r)$ contains the spatial variation and is normalized to $f(0)=1$. 
Because the electric potential is very strong near the nucleus, we can safely neglect atomic binding energies in $f(r)$. In $\mathrm{Pb}$, for example, the potential at the nuclear surface is about $15 \mathrm{MeV}$ compared with valence electron binding energies of a few $\mathrm{eV}$. In addition, to a very good approximation, [1]

$$
\mathcal{N}=R^{-\gamma}
$$

where $\gamma=2\left[1-\sqrt{1-(Z \alpha)^{2}}\right]$ and $R$, often called the equivalent charge radius, is given by

$$
R=\left[\frac{5}{3}<r^{2}>_{\text {charge }}\right]^{1 / 2}
$$

We are not interested in the absolute value of $\mathcal{N}$, but only its variation with nuclear structure. Observable PNC effects are proportional to the matrix element between two atomic states $i$ and $j$

$$
\begin{aligned}
<i\left|H_{P N C, 1}\right| j>= & \frac{G_{F}}{2 \sqrt{2}} C_{i j}(Z) \mathcal{N} \\
& \times\left[-N q_{n}+Z\left(1-4 \sin ^{2} \theta_{W}\right) q_{p}\right] .
\end{aligned}
$$

As mentioned above, this is modified by radiative corrections which we discuss in some detail in section VI. Effects of nuclear structure on PNC are contained in $\mathcal{N}$ and the two quantities

$$
\begin{aligned}
& q_{n}=\int \rho_{n}(r) f(r) d^{3} r, \\
& q_{p}=\int \rho_{p}(r) f(r) d^{3} r .
\end{aligned}
$$

We note that $1-4 \sin ^{2} \theta_{W}$ is a small number; from high energy experiments, $\sin ^{2} \theta_{W}=$ $0.230 \pm .004$. The value of $\sin ^{2} \theta_{W}$ can also be deduced from atomic experiments with an accuracy that will be limited in part by nuclear structure effects, as we discuss in sections VI and VII.

The proton (charge) nuclear form factors needed for $q_{p}$ and $\mathcal{N}$ are generally well known from measurements of the charge distribution of nuclei close to the stable valley and many 
unstable nuclides as well. Neutron nuclear form factors are needed for $q_{n}$, and are not welldetermined experimentally, and statements about them are quite model-dependent. Neutron and proton distributions are often taken to be proportional to each other, scaled by $N$ and $Z$. However, neutron-rich nuclei have larger neutron distributions than the protons and the

reverse is true for proton rich nuclei. In an isotopic sequence, the $A^{1 / 3}$ law is not followed for either the charge or the neutron distributions separately.

\section{B. Experimental Considerations}

As first pointed out by Bouchiat and Bouchiat, [6] the effect of $H_{P N C}$ in neutral atoms grows rapidly with atomic number $Z$, approximately as $Z^{3}$. Thus experimental interest has concentrated on heavy atoms, namely ${ }_{55} \mathrm{Cs},{ }_{81} \mathrm{Tl},{ }_{82} \mathrm{~Pb}$, and ${ }_{83} \mathrm{Bi}$. (For some reviews, see reference [7].) The measured quantity in all experiments is the electric dipole amplitude $\mathcal{E}_{P N C}$ between two electronic states which, in the absence of $H_{P N C}$, would have the same parity and hence would have no electric dipole amplitude connecting them. Denoting the initial and final states by $i$ and $f$, we can write:

$$
\begin{aligned}
\mathcal{E}_{P N C}=\sum_{n}\left[\frac{<f\left|\hat{\mathbf{E}}_{1}\right| n><n\left|H_{P N C}\right| i>}{W_{i}-W_{n}}\right. \\
\left.+\frac{<f\left|H_{P N C}\right| n><n\left|\hat{\mathbf{E}}_{1}\right| i>}{W_{f}-W_{n}}\right]
\end{aligned}
$$

where the first and second terms give the mixing due to $H_{P N C}$ of opposite parity states into the initial and final states respectively. $W$ is the energy of the atomic states, and $\hat{\mathbf{E}}_{1} \equiv-\sum_{j} e \mathbf{r}_{j}$ is the electric dipole charge operator. The magnitude of $\mathcal{E}_{P N C}$ is of order $10^{-9} e a_{0}$ for the heaviest atoms of interest.

Two experimental techniques have evolved for measuring $\mathcal{E}_{P N C}$. One involves applying an external static electric field which, like $H_{P N C}$, mixes in opposite parity states and creates an electric dipole amplitude between the states $i$ and $f$. The interference between this Stark amplitude and $\mathcal{E}_{P N C}$ leads to a parity-violating signature in the optical transition from $i$ to $f$ in which the sign of the interference term reverses with the sense of circular 
polarization of the incident light, and with other vectors specifying the handedness of the experimental arrangement. The other technique uses no external fields, but instead exploits the interference between $\mathcal{E}_{P N C}$ and the magnetic dipole $(M 1)$ amplitude between the same two states. This interference causes parity-violating optical rotation, i.e. a rotation of the plane of polarized light passing through the atomic vapor at wavelengths near the magnetic dipole absorption line.

The Stark interference technique has been used in the all-important measurement of PNC in Cs on the highly forbidden $6 S_{\frac{1}{2}}-7 S_{\frac{1}{2}} M 1$ absorption line at $532 \mathrm{~nm}$, and in the measurement on the $6 P_{\frac{1}{2}}-7 P_{\frac{1}{2}} M 1$ transition in Tl. The optical rotation technique has been applied to the allowed $M 1$ absorption lines at $876 \mathrm{~nm}$ and $648 \mathrm{~nm}$ in $\mathrm{Bi}$, and to the similar $1278 \mathrm{~nm}$ and $1283 \mathrm{~nm}$ lines in $\mathrm{Tl}$ and $\mathrm{Pb}$ respectively; all of which involve transitions among low-lying configurations of $6 p$ electrons, for example $6 P_{\frac{1}{2}}-6 P_{\frac{3}{2}}$ in Tl. Both techniques have reached the 1 percent level of accuracy.

Among the elements studied thus far, $\mathrm{Cs}$ and $\mathrm{Pb}$ are the most likely candidates for comparing different isotopes. It may be possible in the case of Cs to use optical atom traps to carry out measurements on a long string of radioactive isotopes. Measurements on $\mathrm{Pb}$ will probably be restricted to stable or long-lived isotopes. In either case, achieving the level of accuracy discussed in this paper (a few percent down to 1 percent in the isotopic difference), although possible in principle, will be a challenging task in the next generation of atomic PNC experiments.

\section{A SIMPLE MODEL FOR THE NUCLEAR FORM FACTORS}

Given proton and neutron distribution functions, there is no difficulty in calculating $q_{p}, q_{n}$ and the variation in $\mathcal{N}$. In I, we used a simple model to estimate the importance of nuclear structure on PNC observables. We review those results here.

Consider a uniform nuclear charge distribution of radius $R$. This charge produces an electric potential 


$$
V_{c}(r)=Z e^{2} \begin{cases}\left(-3+r^{2} / R^{2}\right) / 2 R, & r<R, \\ -1 / r, & r>R .\end{cases}
$$

A power series for the Dirac wave function inside the nucleus yields

$$
\begin{gathered}
f(r)=1-\frac{1}{2}(Z \alpha)^{2}\left[(r / R)^{2}-\frac{1}{5}(r / R)^{4}+\frac{1}{75}(r / R)^{6}\right] \\
+\mathcal{O}(Z \alpha)^{4} .
\end{gathered}
$$

Again for the sake of simplicity here, we assume that, as for a uniform distribution (for either $n$ or $p$ ), $<r^{4}>=\frac{3}{7} R^{4}$ and $<r^{6}>=\frac{3}{9} R^{6}$ where $R^{2} \equiv \frac{5}{3}<r^{2}>$. From (2.9a) we find (neglecting here any differences between charge and proton radii)

$$
q_{p}=1-0.260(Z \alpha)^{2}+\mathcal{O}(Z \alpha)^{4}
$$

which is insensitive to nuclear structure to this order. From (2.9b), we find

$$
\begin{aligned}
q_{n} & =1-(Z \alpha)^{2}\left(\frac{3}{10} \frac{R_{n}^{2}}{R_{p}^{2}}-\frac{3}{70} \frac{R_{n}^{4}}{R_{p}^{4}}+\frac{1}{450} \frac{R_{n}^{6}}{R_{p}^{6}}\right)+\mathcal{O}(Z \alpha)^{4} \\
& \approx 1-(Z \alpha)^{2}\left(0.038+0.221 \frac{R_{n}^{2}}{R_{p}^{2}}\right)+\mathcal{O}(Z \alpha)^{4}
\end{aligned}
$$

which does depend on the neutron form factor. Here we have introduced equivalent neutron

and proton radii of the form (2.7); the second form in (3.3b) assumes that $\left(R_{n} / R_{p}\right)^{2}-1$ is small.

In this section, we have made rough approximations in order to illustrate the sensitivity of the results to moments of the neutron and proton distributions. For comparison with experiment, a more detailed analysis is necessary, using actual solutions of the Dirac equation for realistic charge distributions and the best available theoretical neutron distributions. This is done in Secs. VI and VII.

\section{INTRINSIC NUCLEON STRUCTURE EFFECTS}

The usual treatment of atomic PNC begins with an effective Hamiltonian for the parity violating electron-nucleus interaction, as in equation (2.2a), which involves normalized proton and neutron distributions 


$$
\begin{aligned}
Z \rho_{p}(\vec{r}) & =\sum_{p}\left\langle\psi_{p}^{\dagger}(\vec{r}) \psi_{p}(\vec{r})\right\rangle, \\
N \rho_{n}(\vec{r}) & =\sum_{n}\left\langle\psi_{n}^{\dagger}(\vec{r}) \psi_{n}(\vec{r})\right\rangle,
\end{aligned}
$$

where $\psi_{N}^{(\dagger)}$ is a destruction (creation) operator for nucleons, and the matrix elements are between nuclear ground states. However, these formulae implicitly assume point-like nature for nucleons, and thus the usual analysis makes no distinction between weak, electromagnetic, or point nucleon distributions, aside from overall charges.

Of course, nucleons do have an internal structure, and this must be properly folded into the above distributions. The internal weak structure is related to, but different from, the electromagnetic structure, and can be calculated in the context of the Standard Model. We demonstrate in this section that the known, electromagnetic structure of nucleons yields a rather small overall effect on atomic PNC calculations, but must be included when extremely high precision results are required.

There has been considerable discussion in recent literature [8,9,10] concerning the possibility of nontrivial strange quark matrix elements in the nucleon. This could lead to a sizable "strangeness radius" of the nucleon, which in turn would modify the weak radius in a well defined way. We allow for this possibility in our analysis, although such a strangeness contribution to atomic PNC is likely to be quite negligible.

In the Standard Model, assuming in addition that strong $\mathrm{SU}(2)$ isospin is a good symmetry for the nucleons, one can extract relations between weak and electromagnetic form factors [11] which then describe the internal nucleon structure:

$$
\begin{aligned}
G_{E}^{\text {weak,p }}\left(q^{2}\right)= & \frac{1}{2}\left(1-4 \sin ^{2} \theta_{W}\right) G_{E}^{\gamma, p}\left(q^{2}\right) \\
& -\frac{1}{2}\left(G_{E}^{\gamma, n}\left(q^{2}\right)+G_{E}^{s}\left(q^{2}\right)\right), \\
G_{E}^{\text {weak,n}}\left(q^{2}\right)= & \frac{1}{2}\left(1-4 \sin ^{2} \theta_{W}\right) G_{E}^{\gamma, n}\left(q^{2}\right) \\
& -\frac{1}{2}\left(G_{E}^{\gamma, p}\left(q^{2}\right)+G_{E}^{s}\left(q^{2}\right)\right) .
\end{aligned}
$$

Here, $G_{E}^{X, N}$ is the usual Sachs electric form factor for a current operator $J_{\mu}^{X}$, where $X$ can represent weak, electromagnetic, or specific quark flavor currents: 


$$
\begin{aligned}
&<p^{\prime}, N\left|J_{\mu}^{X}\right| p, N> \\
& \equiv \bar{u}\left(p^{\prime}\right)\left(F_{1}^{X, N}\left(q^{2}\right) \gamma_{\mu}\right.\left.\quad+i F_{2}^{X, N} \sigma_{\mu \nu} q^{\nu} /(2 M)\right) u(p), \\
& G_{E}^{X}\left(q^{2}\right) \equiv F_{1}^{X}\left(q^{2}\right)+\left(q^{2} / 4 M^{2}\right) F_{2}^{X}\left(q^{2}\right),
\end{aligned}
$$

and

$$
\begin{aligned}
J_{\mu}^{\gamma}= & \frac{2}{3}\left(\bar{u} \gamma_{\mu} u\right)-\frac{1}{3}\left(\bar{d} \gamma_{\mu} d+\bar{s} \gamma_{\mu} s\right) \\
\equiv & \frac{2}{3}\left(J_{\mu}^{u}\right)-\frac{1}{3}\left(J_{\mu}^{d}+J_{\mu}^{s}\right), \\
J_{\mu}^{w e a k}= & \left(\frac{1}{2}-\frac{4}{3} \sin ^{2} \theta_{W}\right)\left(J_{\mu}^{u}\right) \\
& +\left(-\frac{1}{2}+\frac{2}{3} \sin ^{2} \theta_{W}\right)\left(J_{\mu}^{d}+J_{\mu}^{s}\right),
\end{aligned}
$$

are the Standard Model electromagnetic and weak vector currents in terms of quark field operators. (We ignore quarks heavier than strange.) $G_{E}^{s}$ is thus the strangeness electric form factor, and is constrained to be strictly 0 at $q^{2}=0$. Note that one recent estimate 12 gives a strangeness mean-square-radius of around $0.14 \mathrm{fm}^{2}$, roughly as large as that for the neutron electric charge (but of opposite sign). (This quantity can in principle be measured in, e.g. parity violating $\vec{e}^{-}$scattering from nucleons at forward angles.)

With the above relations, we see immediately that at $q^{2}=0$, the usual weak charges are exactly obtained:

$$
\begin{aligned}
Q_{p}^{w} & =\frac{1}{2}\left(1-4 \sin ^{2} \theta_{W}\right), \\
Q_{n}^{w} & =-\frac{1}{2}
\end{aligned}
$$

and one can also predict weak rms radii

$$
\begin{aligned}
\left\langle r^{2}\right\rangle_{I, p}^{w}= & \frac{1}{2}\left(1-4 \sin ^{2} \theta_{W}\right)\left\langle r^{2}\right\rangle_{I, p}^{\gamma}-\frac{1}{2}\left\langle r^{2}\right\rangle_{I, n}^{\gamma} \\
& -\frac{1}{2}\left\langle r^{2}\right\rangle_{I}^{s}+6\left(\frac{1}{2}\right)\left(1-4 \sin ^{2} \theta_{W}\right) /\left(8 M^{2}\right), \\
\left\langle r^{2}\right\rangle_{I, n}^{w}=\frac{1}{2}\left(1-4 \sin ^{2} \theta_{W}\right)\left\langle r^{2}\right\rangle_{I, n}^{\gamma}-\frac{1}{2}\left\langle r^{2}\right\rangle_{I, p}^{\gamma} & -\frac{1}{2}\left\langle r^{2}\right\rangle_{I}^{s}+6\left(-\frac{1}{2}\right) /\left(8 M^{2}\right) .
\end{aligned}
$$


where the subscript I indicates intrinsic nucleon structure, and the last terms in (4.6a) and (4.6b) are the inclusion of the small Darwin-Foldy correction to the radii. Note that the neutron (electromagnetic) contribution to the proton weak radius is not suppressed by any $\left(1-4 \sin ^{2} \theta_{W}\right)$ factor, and thus is surprisingly significant.

Using $\sin ^{2} \theta_{W} \approx .23,\left\langle r^{2}\right\rangle_{I, p}^{\gamma} \approx 0.7 \mathrm{fm}^{2},\left\langle r^{2}\right\rangle_{I, n}^{\gamma} \approx-0.11 \mathrm{fm}^{2},\left\langle r^{2}\right\rangle_{I}^{s}=0$ gives

$$
\begin{aligned}
& \left\langle r^{2}\right\rangle_{I, p}^{w} \approx \frac{1}{2}\left(1-4 \sin ^{2} \theta_{W}\right)\left(2.1 \mathrm{fm}^{2}\right) \\
& \left\langle r^{2}\right\rangle_{I, n}^{w} \approx-\frac{1}{2}\left(.74 \mathrm{fm}^{2}\right)
\end{aligned}
$$

The quantities in parentheses above can be interpreted as the physical size (squared) of the weak distributions. Note that using numbers of $\mathcal{O}( \pm .1)$ [12 for the strangeness radius will have a large effect on $\left\langle r^{2}\right\rangle_{I}^{w}$ for both the proton and (somewhat less so) the neutron.

To a good approximation, considering only rms radii, but no higher moments, the relevant PNC matrix element is then given by a convolution of (point) nucleon centers with their intrinsic structure, yielding a replacement for Eqs. (2.3) and (2.8),

$$
\begin{aligned}
& <i\left|H_{P N C, 1}\right| j> \\
& =\frac{G_{F}}{\sqrt{2}} \int<\left(N Q_{n}^{w} \rho_{n}(\vec{r})+Z Q_{p}^{w} \rho_{p}(\vec{r})\right) \psi_{e}^{\dagger} \gamma^{5} \psi_{e}>d^{3} \vec{r} \\
& =\frac{G_{F}}{\sqrt{2}} C_{i j}(Z) \mathcal{N}\left[N Q_{n}^{w} q_{n}+Z Q_{p}^{w} q_{p}\right],
\end{aligned}
$$

with the quantities $q_{p}$ and $q_{n}$ slightly modified from eqns. (2.9),

$$
\begin{aligned}
q_{(p, n)}=\int d^{3} \vec{r}( & \rho_{(p, n)}^{c}(\vec{r}) \\
& \left.\quad+\frac{1}{6}\left\langle r^{2}\right\rangle_{I,(p, n)}^{w} \nabla^{2} \rho_{(p, n)}^{c} / Q_{(p, n)}^{w}\right) f(r),
\end{aligned}
$$

where $\rho_{p, n}^{c}(\vec{r})$ is now the density distribution of nucleon centers, normalized to 1 .

Assuming, for simplicity, uniform nucleon distributions, with $R_{p} \approx R$,

$q_{p} \approx 1-(Z \alpha)^{2}\left(.26+\frac{.32}{R^{2}}\left(2.1-\left\langle r^{2}\right\rangle_{I}^{s} / 2 Q_{p}^{w}\right)\right)$ 


$$
\begin{aligned}
q_{n} \approx 1-(Z \alpha)^{2}(.038+ & .221 \frac{R_{n}^{2}}{R^{2}} \\
+ & \left.\frac{.32}{R^{2}}\left(.74-\left\langle r^{2}\right\rangle_{I}^{s} / 2 Q_{n}^{w}\right)\right)
\end{aligned}
$$

with all radii measured in fm.

For ${ }^{208} \mathrm{~Pb}$, the internal nucleon structure contributes about 0.002 to $q_{n}$, and a possible strangeness radius discussed above $\left(0.14 \mathrm{fm}^{2}\right)$ would contribute about 5 times less. The internal structure corrects $q_{p}$ by about 0.005 , and the strangeness contribution here would be comparable, about 0.004. In Cs, these numbers turn out to be smaller by about $40 \%$.

From the discussion to come in Sec. VI, we will see that these contributions from (known) finite nucleon structure contributes at about the $0.2 \%$ level in an extraction of the weak nuclear charge when measuring a single isotope of $\mathrm{Pb}(0.1 \%$ level for $\mathrm{Cs})$. This might need to be taken into account in an extremely high precision analysis, but it will not add to the uncertainty in testing the Standard Model. (See also the complete discussion in Sec. VI to compare with the expected scale of nuclear, atomic, and electroweak radiative corrections and uncertainties.) On the other hand, strangeness contributions, which are currently very uncertain, might affect a determination of the weak charge at below the $0.1 \%$ level in $\mathrm{Pb}$, and even less in Cs, and thus are likely to be quite negligible. They could only become relevant if the nucleon strangeness radius were comparable to the electromagnetic radius itself.

In the case of isotopic ratios, the internal nucleon structure plays an even smaller role. This is because errors then come from uncertainties in the difference $q_{n}^{\prime}-q_{n}$ (see Sec. VI). To a good approximation, nucleon structure effects are simply additive in mean square radii and thus cancel in the differences. Thus neither nucleon structure, nor the uncertainties therein, are significant when extracting $\sin ^{2} \theta_{W}$ from isotope ratios. 


\section{NUCLEAR MODELING}

From the rather simplistic model of section III, we already observe that the desired high precision measurements of electroweak parameters will require knowledge of neutron radii in heavy nuclei to within at least several percent. (see also the discussion in section VI) At this level, one clearly must treat higher moments with some care, and the microscopic details of the nucleon distributions may be of some importance. For this reason, we have attempted to evaluate $q_{n}$ and $q_{p}$ numerically, utilizing the best existing nuclear models for neutron, proton, and charge distributions available to us. In this section, we discuss some of the basic features of these models, along with some caveats on their reliability for neutron observables.

The nuclear many-body problem presents a formidable challenge for infinite nuclear matter, and an even greater one for heavy finite nuclei. The most popular route being taken today is some version of Hartree-Fock, which has had considerable success in describing a variety of nuclear properties semiquantitatively.

\section{A. Brueckner-Hartree-Fock}

The underlying basis of nuclear Hartree-Fock calculations is Brueckner theory. The elementary two-body interactions are too strong (especially the short-range repulsion) to lead to meaningful HF calculations. Although there has been extensive work on nuclear matter calculations using Brueckner theory and beyond, for finite nuclei only light ones have been considered [13] and nothing for the nuclei of interest here.

The lowest level is the independent pair approximation. The effective interaction is not $v$ but the Brueckner $G$-matrix, where $G=v F$, with $\psi(1,2)=F(1,2) \phi(1) \phi(2)$. $G$ satisfies a scattering-type equation with a projection operator in intermediate states which excludes scattering back into the Fermi sea; this also results in no phase shift (for pairs in occupied states) and to the two-body wave function "wound" which extends over a "healing distance." 
The $\phi$ 's are to be identified with the HF single quasi-particle functions. We have used $F$ here to denote the two-particle correlation function. For repulsive core potentials, $F$ has a hole (wound) centered about $r=0$. The Brueckner $G$ is non-local, and both energy- and density-dependent.

\section{B. Two-body correlations}

The dependence of the single particle density distribution on the correlation function is relatively small. We can estimate it as follows. Let

$$
\rho_{2}\left(\vec{r}_{1}, \vec{r}_{2}\right)=\rho_{1}\left(r_{1}\right) \rho_{1}\left(r_{2}\right) f\left(\vec{r}_{1}-\vec{r}_{2}\right)
$$

Let $\rho_{1}(r) \propto e^{-r^{2} / a_{N}^{2}}$ and $f(r) \propto e^{-r^{2} / a_{c}^{2}}$ For $a_{c}<<a_{N}$, one finds for the rms size of the single particle density distribution

$$
\begin{aligned}
<r^{2}> & =\int d^{3} r_{1} d^{3} r_{2} r_{1}^{2} \rho\left(\vec{r}_{1}, \vec{r}_{2}\right) \\
& \approx<r^{2}>_{1}\left[1+\frac{1}{4 \sqrt{2}}\left(\frac{a_{c}}{a_{N}}\right)^{3}\right]
\end{aligned}
$$

where $\left\langle r^{2}>_{1}\right.$ corresponds to $\rho_{1}$. For heavy nuclei (say $a_{N} / a_{c} \approx 7.0 / 0.7 \mathrm{fm}$ ), the correction is less than $2 \times 10^{-4}$, which is below our level of current concern.

\section{Phenomenological Hartree-Fock, including deformation and pairing}

Because of the numerical complexity, most HF calculations have employed phenomenological potentials intended to simulate the Brueckner $G$-matrix. The most commonly used potentials are varieties of the very convenient Skyrme interaction. The Skyrme interactions are of the delta-function form and as such lead to single particle equations with local onebody potentials and spatially-dependent effective masses, with no more complication than Hartree calculations. In contrast, finite range interactions lead to non-local single particle

potentials arising from the exchange term. Momentum-dependent Skyrme interactions do not lead to further complications and simulate some effects of finite range. Calculations 
have also been done with finite range forces, using e.g. the Gogny interaction [14 Note that none of these phenomenological potentials are intended to reproduce free nucleon-nucleon scattering. There are of the order of eight (more or less) adjustable parameters in any model. 15.

Most nuclear structure calculations on heavy nuclei are carried out in the deformed Hartree-Fock or the Hartree-Fock-Bogolyubov approximations. The latter include BCS-type pairing. Hartree-Fock encompasses a limited class of correlation structure. Only correlations of a collective nature are included. It is not surprising to find that in HF neutron and proton densities tend to track one another. Nevertheless, they do exhibit the expected behavior that the neutron rms radius increases more rapidly than the proton one with increasing $A$ in an isotopic sequence. The relative tracking (variation in the neutron skin) depends on the way in which symmetry energy is handled.

Intrinsic deformations play a key role in spherically averaged proton and neutron densities. In the uniform, incompressible approximation, for example, the mean square radius is increased according to 16

$$
<r^{2}>_{\beta}=<r^{2}>_{0}\left[1+\frac{5}{4 \pi}<\beta^{2}>\right]
$$

where $\beta$ is the nuclear shape parameter, proportional to the quadrupole moment. $\beta$ can attain values of the order of $1 / 3$ and changes in $<\beta^{2}>$ among isotopes can produce deviations in spectroscopic isotope shifts by an order of magnitude from the $A^{1 / 3}$ law. Although HF calculations tend to yield spherical $(\langle\beta=0>$ ) or near spherical equilibrium shapes for the $\mathrm{Pb}$ isotopes, $<\beta^{2}>$ is not zero and changes in $<\beta^{2}>$ have been considered by some authors.

\section{Relativistic Mean Field}

Although nuclear structure is primarily nonrelativistic, considerable success has been achieved by treating the nucleons and protons as point Dirac particles [17] [see, however, Achtzehnter and Wilets [18]] interacting with phenomenological vector and scalar mesons in 
the mean field approximation. The vector mesons can be identified with the isoscalar omega and the isovector rho mesons; the scalar meson is a simulation of two-pion exchange. The mesons are treated in the mean field, or c-number approximation. An attractive feature of the model is that the strong spin-orbit potential appears to emerge "naturally." While this turns out to be true for isoscalar potentials, it fails badly for the isovector potentials, but can always be parameterized to yield reasonable results. [18]

In order to fit nuclear properties, it has been necessary to go beyond linear field theory. Self-interaction of the scalar field has been introduced, with additional parameters. Among other problems, this solved the compression modulus anomaly, which is much too large in the linear model. The total number of adjustable parameters which must be introduced is comparable to that required in models using Skyrme forces. As with Skyrme forces, the mean field approximation does not lead to nonlocality in the one-body potentials.

\section{E. RPA and MCHF}

The tail of the neutron or proton distribution has correlation/polarization corrections not described by HF, at least for large distances. The reason for this is that the individual nucleon wave functions see the potential of the "mean" self-consistent core. In the tail region, the residual core tends to relax. This is most evident for the separation energy: In $\mathrm{HF}$, the separation energy of a nucleon is just the energy eigenvalue (Koopmanns' theorem) if the core is frozen. If the energy of the residual nucleus is recalculated self consistently, the

separation energy is reduced by what is termed the rearrangement energy. Correlations of this type are included through RPA, which is equivalent to small amplitude, time-dependent Hartree-Fock.

Other types of correlations could be included through multiconfigurational Hartree-Fock, which, as the name implies, means that the trial wave function is not a single determinant, but a sum of determinants (configurations). This serves two purposes: correlations of the kind allowed by the choice of configurations are included, and the occupation of these con- 
figurations modifies the mean field potential and single particle functions.

\section{F. Beyond Hartree-Fock plus}

Most HF and HFB calculations do reasonably well in reproducing atomic isotopic shifts for the even-even isotopes of PB below 208. So, incidentally, does the droplet model of Meyers [19]. They all fail to reproduce even-odd staggering, which shows odd nuclei to be smaller than the mean of their even-even neighbors, and also do badly on the shifts above 208.

There are no giant shell model diagonalization calculations available which yield densities

for heavy nuclei. Such would be very valuable for comparison with Hartree-Fock results, since in principle they include all types of correlations, limited only by the size of the basis.

An idealized shell model calculation should be based on realistic two body interactions, the kind which fit free two-body scattering data. The Hilbert space could be divided into a "near" and a "far" space. The effective two body interaction could be obtained by solving for the Brueckner $G$-matrix with the intermediate states excluded from the near space. The far space scattering states could be approximated by plane waves if the momentum sphere separating the spaces is sufficiently large. [13] The Hamiltonian matrix for the inner space, using the effective interaction, is then diagonalized.

\section{G. Summary and Discussion}

Unfortunately, not all of the theoretical considerations discussed above have been incorporated in any single calculation. Heavy nuclei pose a difficult challenge for reliable, detailed modeling at the level of precision we require. There do exist in the literature a number of recent efforts, as discussed in sections $\mathrm{C}$ and $\mathrm{D}$ above, which involve either relativistic or non relativistic Hartree-Fock nuclear calculations. We have accumulated densities from several of these authors in order to evaluate $q_{p}$ and $q_{n}$ and make comparisons among the different 
models. These include various HF calculations with Skyrme forces, [20,21] an HFB calculation with a Gogny finite range D1S interaction, [14,22 and several relativistic mean field models. 23, 24] The results are presented in Sec. VII.

The modelers fit their adjustable parameters to choices among various bulk properties (energy per nucleon, compressibility modulus, symmetry energy, etc.), and properties of particular nuclei (energies, charge radii, deformations, spectra, multipole sum rules, etc.) Indeed, the physics behind the models comes, in part, from the choice of the particular observables included in the parameter fits. The models we have selected all do roughly equally well in fitting the wide range of nuclear observables available across the periodic table. [21,23]

The analysis of atomic PNC, as discussed in sections II and III, relies on a detailed knowledge of neutron distributions in nuclei. The lack of unambiguous, precise experimental measures of neutron radii means that all of these models must "extrapolate" to the desired neutron properties. Charge radii, on the other hand, are in a certain sense "built in", in that the set of observables to which the nuclear model parameters are fit includes charge radii of several even-even nuclei, one of which is ${ }^{208} \mathrm{~Pb}$. In defense of the models, they predict with good success the charge radii of other even-even nuclei not included in the fit, [24] and also reproduce the well-measured isotopic charge radius shifts for, e.g. the even isotopes of Pb. [20,37] However, they do not reproduce the observed even-odd staggering of charge radii very well, nor are the results as good for the charge radius of ${ }^{210} \mathrm{~Pb}$, an indication that some care should be taken when considering non closed-shell cases.

There do exist some data which may give more direct information on the neutron skin. This might be used as additional input to these nuclear models, and could help further constrain the predictions for neutron radius, and neutron isotope shifts, if one could demonstrate consistency in the results. Perhaps the best known data comes from $800 \mathrm{MeV}$ polarized proton scattering from ${ }^{208} \mathrm{~Pb}$. [30,31] This gives $R_{n}-R_{p}=0.14 \pm 0.04 \mathrm{fm}$. The quoted error, which is quite small for our purposes, contains both statistical and certain theoretical uncertainties as stated. However, there are still additional theoretical uncertainties, involving e.g. 
assumptions about the in medium nucleon-nucleon t-matrix, and the result seems to exhibit a rather large and troubling energy dependence. [32] The absolute value of $R_{n}$ in one isotope is believed to be fairly difficult to obtain with confidence using such experiments. However, it may be that the relative shift in $R_{n}$ among isotopes involves cancellations that reduce these theoretical uncertainties. Measurements on other $\mathrm{Pb}$ isotopes (data [33] apparently exists for ${ }^{206} \mathrm{~Pb}$ ) would clearly be of interest in this context. Further experimentation and theoretical analysis at other energies are also crucial to demonstrating the consistency of the results.

Experiments involving intermediate energy charged pion scattering from nuclei may also help further constrain the neutron radii, or the relevant isovector model parameters, as well. Such data exists for ${ }^{208} \mathrm{~Pb}$, [34] but again the absolute normalization poses a real challenge to analyses. [31] Taken at face value, the $\vec{p}$ and $\pi$ results for lead neutron radii do agree with one another reasonably well, and also match with e.g. the Gogny finite range HartreeFock calculations. Another experimental possibility involves energies and sum rule strengths of giant multipole resonances. [35] The uncertainties here are even larger, and difficult to estimate. Clearly, a reliable set of such additional "strong probe" inputs, including yet other options such as $\alpha$ particle scattering, kaon scattering, Coulomb displacement energies, etc., could be an aid in constraining the theoretical models on neutron properties.

Another promising experimental possibility for the future might be direct electroweak experiments, such as parity violating asymmetries in elastic, intermediate energy $\vec{e}^{-}-$nucleus scattering, as proposed by Donnelly, Dubach, and Sick, [36] or perhaps elastic $\nu$ scattering. The reactions and analyses are quite clean, just as in the charge scattering case. There would be, for example, no serious ambiguities in the absolute scale of the radii measured. Such experiments would in fact be sensitive to the full nuclear weak charge distribution, rather than just the RMS radius, which many of the strong probe measurements are primarily sensitive to. Because such experiments could be done at moderate momentum transfers $\left(q \sim 1 \mathrm{fm}^{-1}\right.$ ), the extraction of nuclear distribution information would be much less sensitive to the precise values of electroweak parameters than in the corresponding atomic parity 
violation case. The asymmetries and $\nu$ cross sections are naturally extremely small, and the experimental challenges are formidable. Nevertheless, recent estimates for the parity violating asymmetries indicate that measurements sensitive e.g. to the neutron RMS radius in ${ }^{208} \mathrm{~Pb}$ at the $1 \%$ level are feasible. [36] As we will see in sections VI and VII, such a level would make the nuclear structure uncertainties quite negligible for the purposes of extracting standard model parameters from single isotope atomic PNC measurements.

In any case, current model fitting has been done with the best and most reliable data at hand, most of which are not directly sensitive to neutron distributions. It is always difficult to estimate the theoretical uncertainties in such model calculations. In this section, we have already mentioned several potentially important missing features that future work should address, especially involving nucleon correlations. We have not attempted here to try to choose a "best model" from the various ones we examined, but rather wish to evaluate the existing spread in predictions as an effective lower bound on the theoretical uncertainties involved. One might, however, try to make a selection based on detailed comparisons, specifically targeting a good fit to heavier nuclei energies, isotopic shifts, giant dipole properties, and other quantities potentially sensitive to isovector properties. We encourage work in such directions. The goal should be to find the most reliable model(s) while still retaining an estimate of the remaining theoretical uncertainties.

\section{ERROR ANALYSIS AND TESTS OF ELECTROWEAK PHYSICS}

One of the motivations for further improving atomic parity violation experiments is to test the Standard Model at the level of its one-loop electroweak radiative corrections. This allows one to probe for possible small "new physics" effects, which would appear as further loop corrections or more directly as additional interactions at the tree level (i. e. without loop corrections). A good example of the latter, to which atomic PNC is particularly sensitive, is exchange of a second, more massive, neutral Z-boson required in theories with larger gauge groups. In the Standard Model, the loop contributions are separated into two 
parts: fixed radiative corrections due to contributions from the known quarks, leptons and bosons, and the heavy physics part due to contributions from the top quark and the Higgs boson. One is interested in an experimental determination of the heavy physics part, which in the language of Marciano and Rosner [25] is expressed in terms of weak isospin-conserving, $\mathrm{S}$, and isospin-breaking, T, effects. These two constants [26] are a convenient way not only of including uncertainties in the top quark and Higgs masses, but of parameterizing the effects of some specific classes of new physics as well. It turns out that low energy PNC measurements are nicely complementary to high energy measurements such as direct Zboson production, since both the radiative corrections and the sensitivities to new tree-level interactions are quite different.

To show how the radiative corrections and the possible new physics enter into atomic PNC, and how they might compare in size to nuclear structure effects, we begin by rewriting equation (2.9) in the form:

$<i\left|H_{P N C, 1}\right| j>=\frac{G_{F}}{2 \sqrt{2}} C_{i j}(Z) \mathcal{N}\left[Q_{W}(N, Z)+Q_{W}^{n u c}(N, Z)\right]$

where $Q_{W}(N, Z)$, known as the nuclear weak charge, is the quantity of primary interest to electroweak theory, and in the standard model without radiative corrections reduces to:

$$
Q_{W}^{0}=-N+Z(1-4 \bar{x})
$$

where $\bar{x} \equiv \sin ^{2} \theta_{W} \cdot Q_{W}(N, Z)$ is determined from atomic experiments by combining atomic measurements of $\left\langle i\left|H_{P N C, 1}\right| j>\right.$ with calculations of both atomic structure (contained in the factor $C_{i j}$ ) and nuclear structure. The nuclear structure corrections are contained in $Q_{W}^{n u c}(N, Z)$, which is given by:

$$
\begin{aligned}
Q_{W}^{n u c}(N, Z) & \equiv Q_{W}\left(\left[q_{n}-1\right] N,\left[q_{p}-1\right] Z\right) \\
& \approx-N\left(q_{n}-1\right)+Z(1-4 \bar{x})\left(q_{p}-1\right)
\end{aligned}
$$

Nuclear structure is also contained in the normalization $\mathcal{N}$, but as we will see in section VII, $\mathcal{N}$ is determined by the nuclear charge distribution, which is usually known experimentally. 
When we include possible new physics, together with the effects of radiative corrections which have been calculated by others, [25] $Q_{W}(N, Z)$ becomes:

$$
\begin{aligned}
& Q_{W}(N, Z)=(0.9857 \pm 0.0004)(1+0.00782 T) \\
& \times[-N+Z(1-(4.012 \pm 0.010) \bar{x}] \\
&+Q_{\text {tree }}^{\text {new }}(N, Z)
\end{aligned}
$$

where $\bar{x}$ is assumed here to be defined at the mass scale $m_{Z}$ by modified minimal subtraction, 25] and is given by:

$$
\bar{x}=.2323 \pm .0007+.00365 S-.00261 T .
$$

The errors indicated in (6.4) and (6.5) come from uncertainties both in experimental input parameters and in evaluations of known physics loop-diagrams. The unknown, heavy physics loop-corrections are contained in the parameters $S$ and $T$, which depend upon the heavy masses, and are defined such that $S=T=0$ if $m_{H}=100 \mathrm{GeV}, m_{t}=140 \mathrm{GeV}$, and if there is no new physics beyond the Standard Model. Including $Q_{\text {tree }}^{\text {new }}(N, Z)$ in $Q_{W}(N, Z)$ allows for additional tree-level physics beyond the Standard Model. For example, exchange of the extra $Z_{x}$ in $\mathrm{SO}(10)$ models 25,27] (assuming no $Z_{x}-Z$ mixing) would make:

$$
Q_{\text {tree }}^{\text {new }}(N, Z) \approx 0.4(2 N+Z) m_{W}^{2} / m_{Z_{\chi}}^{2} .
$$

It is useful to consider how well the parameters in $Q_{W}$ are currently known. The central value of $Q_{t r e e}^{\text {new }}(N, Z)$, determined mainly by Cs PNC measurements, is about $2.2 \pm 1.6 \pm .9$ (if all other heavy physics in equation (6.4) is ignored), and corresponds in the $\mathrm{SO}(10)$ model to $m_{Z_{\chi}} \approx 500 \mathrm{GeV}$. Conversely, assuming no new tree-level physics (i.e., $Q_{\text {tree }}^{\text {new }}=0$ ), the experimental uncertainty in $\mathrm{T}$ is currently around \pm 1 , and in $\mathrm{S}$ around \pm 3 , the latter determined largely from Cs PNC. Ultimately, as Marciano and Rosner have indicated, an effort to reduce the uncertainty in $\mathrm{S}$ to \pm 0.2 is extremely important, since at that level it is sensitive even to minimal one-doublet technicolor models. This sort of accuracy is an extreme challenge to either high energy or atomic experiments. Current knowledge of $\sin ^{2} \theta_{W}$ from 
a global analysis of electroweak data [28] can be summarized by $\bar{x}=0.230 \pm .004$, (roughly $2 \%$ uncertainty). If future high energy measurements were to reduce the uncertainty in $\bar{x}$ beyond what is attainable in atoms, the atomic experiments would still be valuable for improving the limits, e.g., on an additional Z.

In summary, any improvement in determining atomic PNC is likely to provide useful information about electroweak physics, and it becomes extremely important to work out how much nuclear structure uncertainties may be a limiting factor, and to reduce these uncertainties where possible.

We first consider the impact of nuclear uncertainties on PNC measurements of single isotopes. PNC experiments to date have been done on stable isotopes of heavy atoms, namely $\mathrm{Cs}, \mathrm{Pb}, \mathrm{Bi}$, and $\mathrm{Tl}$, and have not compared different isotopes of the same element. From equation (6.1) we derive an expression for the uncertainty in $Q_{W}$ in terms of the uncertainties in atomic and nuclear structure and in the measured quantity $\mathcal{O} \equiv<i\left|H_{P N C, 1}\right| j>$ :

$$
\frac{\delta Q_{W}}{Q_{W}} \approx \frac{\delta \mathcal{O}}{\mathcal{O}}-\frac{\delta C_{i j}}{C_{i j}}-\frac{\delta \mathcal{N}}{\mathcal{N}}-\frac{\delta Q_{W}^{n u c}}{Q_{W}}
$$

If we assume that $\mathcal{O}$ can be measured to arbitrary accuracy, and that proton distributions (which will influence $\delta \mathcal{N}$ ) are also well enough understood and/or measured, there remain the uncertainties coming from atomic and nuclear structure, which we can write in the form:

$$
\frac{\delta Q_{W}}{Q_{W}} \approx-\frac{\delta C_{i j}}{C_{i j}}-\delta q_{n}
$$

where we have dropped all terms containing the factor $1-4.012 \bar{x}$, which should be quite negligible due to the accidental value of $\bar{x} \approx \frac{1}{4}$. Rewriting in terms of the weak interaction parameters, we obtain:

$$
0.014 \frac{Z}{N} \delta S+\frac{\delta Q_{\text {tree }}^{\text {new }}(N, Z)}{Q_{W}} \approx-\frac{\delta C_{i j}}{C_{i j}}-\delta q_{n}
$$

ignoring the contribution of the weak-isospin breaking parameter $\mathrm{T}$ which cancels to better than $10 \%$ for the full range of $Z / N$ found in the elements of experimental interest. Thus a PNC measurement in a single isotope can set limits on the weak-isospin conserving parameter 
$S$ and/or new tree-level interactions, and in fact the best limits on both of these parameters now come from PNC measurements in atomic cesium. To determine the role of nuclear structure, we must compare the uncertainty $\delta q_{n}$ on the right hand side of equation (6.9) to the atomic structure uncertainty $\delta C_{i j} / C_{i j}$. This we do later, in section VII.

Because of the difficult atomic physics calculations, there has been some serious interest in measuring parity violation in a chain of isotopes. Taking ratios between isotopes cancels essentially all dependence on atomic structure. Unfortunately, although the atomic physics indeed cancels in the ratio, the nuclear structure does not. Referring to equation (6.1) we consider the ratio:

$$
\mathcal{R} \equiv \frac{\mathcal{O}}{\mathcal{O}^{\prime}}=\frac{\left[Q_{W}(N, Z)+Q_{W}^{n u c}(N, Z)\right] \mathcal{N}}{\left[Q_{W}\left(N^{\prime}, Z\right)+Q_{W}^{\text {nuc }}\left(N^{\prime}, Z\right)\right] \mathcal{N}^{\prime}}
$$

where primed and unprimed quantities refer to different isotopes. The sensitivity of $\bar{x}$ and $Q_{t r e e}^{\text {new }}(N, Z)$, extracted from this ratio, to the nuclear structure is then given approximately by

$$
\begin{aligned}
Z \frac{\delta \bar{x}}{\bar{x}} & -\delta Q_{\text {tree }}^{\text {new }}(N, Z)+N \frac{\delta \Delta Q_{\text {tree }}^{\text {new }}}{\Delta N} \\
& \approx \frac{N N^{\prime}}{\Delta N}\left[\frac{\delta \mathcal{R}}{\mathcal{R}}+\frac{\delta(\Delta \mathcal{N})}{\mathcal{N}}+\frac{\delta\left(\Delta q_{n}\right)}{q_{n}}\right] .
\end{aligned}
$$

where we have made simplifying assumptions that the isotopes are close together, i.e. $\Delta N \equiv$ $N^{\prime}-N<<N$, that $\sin ^{2} \theta_{W} \approx 1 / 4$, and where we have used e.g. $\left(\delta q_{n} / q_{n}-\delta q_{n}^{\prime} / q_{n}^{\prime}\right) \approx$ $\delta\left(\Delta q_{n}\right) / q_{n}$, which is numerically accurate for the models of $\mathrm{Pb}$ we have considered. Because of the special sensitivity of atomic PNC to any additional heavy Z-bosons, we note as an example that a determination of $m_{Z_{x}}$ in the model of equation (6.6) would be constrained by replacing the left hand side of equation (6.11) by $\left(Z \delta \bar{x} / \bar{x}-0.4 Z \delta\left(m_{W}^{2} / m_{Z_{x}}^{2}\right)\right)$.

The uncertainties on the right side of (6.11) are effectively in the relative difference between quantities for two isotopes. In principle, different nuclear models which disagree on the absolute values of, say, $q_{n}$ may agree on the relative change in this quantity to a much higher degree of accuracy. However, such a reduction in uncertainty in the terms within the brackets in expression (6.11) is roughly compensated by the factor $N / \Delta A$. Comparing with 
equation (6.9) for a single isotope, in which any new tree-level interactions enter with the equally uncertain loop-parameter $S$, we see that when we instead compare isotopes, $Q_{\text {tree }}^{\text {new }}$ appears together with a different parameter, $\bar{x}$, which is independently measurable in high energy experiments.

\section{DISCUSSION}

To calculate $q_{n}$, we use various theoretical predictions of neutron and proton distributions from the literature. Proton distributions are used to compute $f(r)$, the electronic wave function overlap defined in equation (2.5). We solve numerically for single electron Dirac $s_{1 / 2}$ and $p_{1 / 2}$ wave functions near the origin, in the Coulomb potential of the nuclear charge distribution (as discussed in section II), and make no approximation of a power series in $Z \alpha$, as was done e.g. for equation (3.2). We have neglected the contributions to the nuclear charge distribution from internal neutron structure, as discussed in section IV. We estimate the error associated with this assumption to be well below the level of the model uncertainties themselves. The quantity $q_{n} \equiv \int \rho_{n}(r) f(r) d^{3} r$ is then calculated directly from the corresponding neutron distribution.

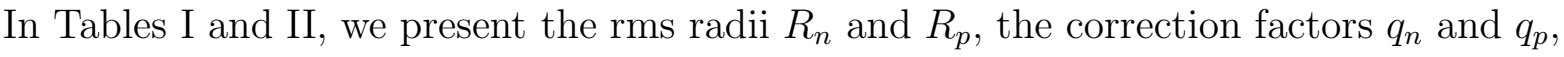
and the electron normalization $\mathcal{N}$, for several nuclear models of the $\mathrm{Pb}$ isotopes 202 and 210.

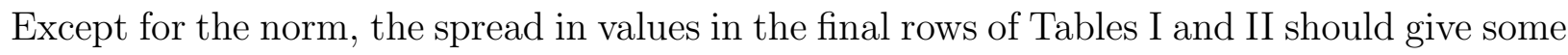
indication of a lower limit on the current level of theoretical model-dependent uncertainties, assuming that one accepts these models as equally phenomenologically reasonable.

The normalization factor $\mathcal{N}$ defined in equation $(2.5)$, which is proportional to $1 / f(r \gg$ $\left.R_{p}\right)$, is defined arbitrarily here as $0.10361 / f(300 \mathrm{fm})$. The numerator, $f^{\operatorname{expt}}(300 \mathrm{fm})$, is evaluated using a model independent experimental charge distribution from electron scattering off ${ }^{208} \mathrm{~Pb}$. As stated earlier, we are not concerned with the absolute value of the norm, but only its dependence on atomic weight and charge distribution. This definition simply scales $\mathcal{N}$ to be near 1.0. For ${ }^{208} \mathrm{~Pb}$, the model spread in the normalization from Table $\mathbb{1}$ might 
appear to contribute at a significant level. One can, however, consider correcting $\mathcal{N}$ by using an approximate formula relating $\mathcal{N}$ to the charge radius, namely $\mathcal{N}=R^{-\gamma}$, as in eqn. (2.6). This is given by

$$
\mathcal{N}^{\prime}=\mathcal{N}\left(R^{\gamma} / R_{\text {expt }}^{\gamma}\right)
$$

The model spread in this $\mathcal{N}^{\prime}$ is significantly reduced. The point is that these models are not precisely reproducing the observed charge radii of the lead isotopes, which feeds rather directly into a calculation of $\mathcal{N}$. The correction factor above compensates for this, using the existing high precision measurements of charge radii from optical isotope shifts and electron scattering. 37.38

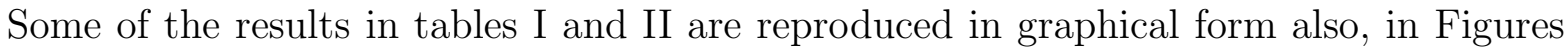
1, 2, and 3. In Fig. 1, we plot the predicted $R_{n}$ versus atomic weight for several even lead isotopes, and in Fig. 2 the ratio $R_{n} / R_{p}$. The spread in $R_{n}$ among models is decidedly larger than the spread in $R_{p}$. We do note a systematically larger neutron radius in the relativistic models. [29] The origin of this is indeed not yet completely understood, but may be connected with larger asymmetry energies found in these models. This in turn might tend to pull neutron and proton distributions together where the densities are high, leaving a somewhat larger neutron tail.

In Fig. 3, we plot $q_{n}$ versus atomic weight for several even lead isotopes. The spread is closely related to the spread in $R_{n} / R_{p}$ shown in Fig. 2, as might be expected from the simplified formulas (1.1) or (3.3b) based on uniform nuclear charge density. Estimates of $q_{n}$ using these simplified formulas yield the same general trends as the detailed calculations, with absolute values differing generally by parts in a thousand or less. The relativistic models yield somewhat smaller $q_{n}$, due to their larger $R_{n} / R_{p}$ ratio.

In the case of single isotopes, the total nuclear model spread does not appear to be the most serious problem in using equation (6.8) or (6.9) to extract weak interaction parameters from atomic PNC . For ${ }^{208} \mathrm{~Pb}$, the typical full spread in calculated $q_{n}$ is $\lesssim 0.005$. In the case of ${ }^{133} \mathrm{Cs}$, the sensitivity to nuclear structure is even weaker, due to the smaller value of $(\mathrm{Z} \alpha)$. 
A larger uncertainty, at least at the present time, is due to atomic physics calculations. [2.]3 For example, Cs is one of the most favorable elements from the point of view of atomic theory, and to achieve the current level of quoted uncertainty of $\delta C_{i j}(Z) / C_{i j}(Z) \approx 1 \%$ in Cs is an impressive task. But this uncertainty is still probably larger than the uncertainty in $q_{n}$ for Cs. Significant future improvement in atomic calculations is likely to be difficult. Thus, aside from any experimental uncertainties, atomic structure is the present limiting factor in getting $Q_{W}$ and the associated weak parameters from single isotope atomic PNC measurements, and appears to remain so even after considering the possible nuclear physics effects. This conclusion is consistent with the findings mentioned in the calculation of reference 2 .

Consider next the ratios in an isotopic chain, for example $\left({ }^{202} \mathrm{~Pb} /{ }^{208} \mathrm{~Pb}\right)$. Referring to equation (6.11) we see that the PNC experiments would then be measuring $\bar{x}$ and/or observing new tree level physics. For definiteness let us assume no new tree level physics. Then a $\pm 1 \%$ extraction of $\bar{x}$ would require $\delta \Delta q_{n} \lesssim 6 \cdot 10^{-4}$, and $\delta \Delta \mathcal{N} / \mathcal{N} \lesssim 6 \cdot 10^{-4}$. Assuming uniform nuclear distributions, this implies $\delta \Delta\left(R_{n} / R_{p}\right) \lesssim 4 \cdot 10^{-3}$. Referring to Table III, which shows the change in various quantities between these two particular lead isotopes, the model spread for $\delta \Delta q_{n}$ is around $9 \cdot 10^{-4}$ and for $\delta \Delta \mathcal{N} / \mathcal{N}$ is about $6 \cdot 10^{-4}$. Note however that when $\mathcal{N}$ is corrected as in (7.1) above, using experimental knowledge of charge radii, this spread, at least, is significantly reduced to below the $\pm 1 \%$ level. This is seen from the final column in Table [11. But the spread in $\Delta q_{n}$ remains, and is comparable to the accuracy needed for a $1 \%$ extraction of $\bar{x}$. Similarly, the model spread in $\left(R_{n} / R_{p}\right)$ from Table III is about $5 \cdot 10^{-3}$, which likewise corresponds to a $>1 \%$ spread in $\bar{x}$.

If we exclude the relativistic models, which seem to have substantially different neutron radii from the conventional H-F calculations, the model spread just among the various Skyrme parameterizations considered gives $\delta \Delta q_{n} \approx 4 \cdot 10^{-4}$. It thus appears unlikely that $\mathrm{PNC}$ measurements comparing $\mathrm{Pb}$ isotopes could yield much better than a $1 \%$ determination of $\bar{x}$, unless there is significant improvement in understanding of nuclear structure.

The same results can be seen perhaps more clearly in Fig. 因, which displays in graphical 
form the values of $\Delta q_{n}(202 \rightarrow 208)$ from Table III versus the different models considered. The spread in predictions of this quantity is actually larger than 100\%. Also shown in the figure is a typical scale of $1 \%$ in the weak angle. As noted already, the model spread is too large for extractions of $\bar{x}$ at the sub $1 \%$ level if one cannot otherwise eliminate or improve any of the models used. On the other hand, the nuclear structure uncertainties may not preclude a significant improvement in sensitivity to new Z bosons or other new tree-level physics in equations (6.11), particularly if $\bar{x}$ is determined well by high energy experiments.

Although the nonrelativistic models do appear to cluster together somewhat, one should perhaps be a bit wary of their apparent self-consistency. For example, a modification of the coefficient of the isovector (n-p asymmetry) surface term, a $\left(\rho_{p}-\rho_{n}\right) \nabla^{2}\left(\rho_{p}-\rho_{n}\right)$ term in the Skyrme Lagrangian, 21] has little effect on most bulk properties, and hence on the goodness of the Skyrme fits. 23 This term, however, does modify the neutron skin significantly. Reinhard's rough estimates show that an uncertainty of $\Delta R_{n} \approx \pm .15 \mathrm{fm}$ is not unreasonable. [23] This in turn can modify the quantity shown in Fig. 14 by amounts of $\mathcal{O}\left(6 \cdot 10^{-4}\right)$, larger than the spread in the given Skyrme models. The relativistic models do not have such flexibility, as the isovector rho couplings are largely constrained by isotopic trends in ground state energies and charge radii, but this is of course no guarantee that these models correctly describe all isovector properties equally well.

In the case of Cs isotopes, accurate calculations for neutron radii (or even proton radii) are difficult. They have odd Z, and require additional approximations to deal with unfilled shells, as well as deformations. The lack of success in predicting the even-odd staggering of $\delta\left\langle r^{2}\right\rangle_{c h}$ in lead isotopes indicates the seriousness of these problems. An estimate of the scales involved, however, can be made using calculations with existing nuclear codes. One such result 39 gives $R_{n} / R_{p} \approx 1.03$ for ${ }^{135} \mathrm{Cs}$, and $\Delta R_{n} / R_{p}\left({ }^{131} \mathrm{Cs} \rightarrow{ }^{135} \mathrm{Cs}\right) \approx 5 \cdot 10^{-3}$. If this latter number itself has a 100\% uncertainty (for comparison, see Fig. 14 for the case of lead which does show a $100 \%$ spread among model predictions of the equivalent quantity $\Delta q_{n}$ for about the same $\Delta A / A)$, then the uncertainty in $\bar{x}$ from this fairly small range of isotopes would be approximately $1 \%$. $\Delta A$ of up to 10 or higher may be experimentally possible for 
Cs, which might help to reduce the nuclear physics uncertainties. From the experimental side, the absence of stable isotopic partners to ${ }^{133} \mathrm{Cs}$ makes it difficult to obtain values of $\Delta R_{n} / R_{p}$ from parity violating electron scattering, or $\vec{p}$ elastic, or pion experiments, as may be possible for the lead isotopes. Further work on theoretical estimates for Cs isotopic radii is clearly called for.

Given a set of experimental results for isotopic PNC ratios, one can also consider a bootstrap procedure: from atomic experiments over several isotope differences, use the various models to extract the weak mixing angle. Then, only those models which yield the same $\sin ^{2} \theta_{W}$ for the various isotopic pairs are acceptable. Unfortunately, the various nuclear models we have considered (for lead isotopes near ${ }^{208} \mathrm{~Pb}$ ) yield predictions for the PNC ratios which are fairly linear with $\Delta A$. Since this prediction is also roughly linear with $\sin ^{2} \theta_{W}$, it appears that the various nuclear models could be internally consistent, each yielding a unique $\sin ^{2} \theta_{W}$ but differing from model to model about the extracted value. Of course, one cannot draw any firm conclusions about this until after the data are known. There are indeed some slight deviations from linearity, especially for non-closed shell isotopes, and one may be able to take advantage of this. In essence, this bootstrap idea uses PNC atomic isotope ratios themselves as our desired additional constraint on neutron properties - with a large enough set of PNC data, one could hope to simultaneously constrain the nuclear model parameters and measure the weak mixing angle.

\section{CONCLUSIONS}

For the case of $\mathrm{Pb}$, in order to extract electroweak parameters from atomic PNC experiments at a level of precision which would be considered "significant" for testing the Standard Model, we have shown that it is necessary to have confidence in the isotopic relative neutron/proton radius shift, $\Delta\left(R_{n} / R_{p}\right) / \Delta A$, to better than a few times $10^{-4}$. We have examined various nuclear model calculations, and find that the spread in theoretical

values corresponds to an uncertainty in the weak mixing angle greater than $1 \%$, with the 
assumption that no new physics is present. Without some further basis for discriminating among the various models, the spread represents a lower bound to the uncertainties in the calculated values.

The basic problem is essentially that the models have been parameterized to fit properties like charge distributions, which are not directly sensitive to neutron distributions. As Reinhard has shown, it appears that a surface symmetry energy term in certain nonrelativistic (Skyrme interaction) nuclear models can be "dialed" somewhat to change the neutron size without significantly spoiling the basic fits. Including data which are more sensitive to neutron properties, such as isotopic trends in ground state properties, and perhaps giant resonance energies and sum rules, could be useful to constrain such terms.

There do exist experiments which are sensitive to neutron radii, e.g. $\pi^{+} / \pi^{-}$scattering, and medium energy polarized proton scattering. If the quoted errors on the latter can be taken literally, one could use it to discriminate among the various models and provide the confidence one needs to extract the desired electroweak parameters from atomic experiments. It would be valuable to repeat the experiments and analyses at other energies in order to demonstrate the consistency of the results, and to consider both $\pi^{ \pm}$and $\vec{p}$ scattering on multiple $\mathrm{Pb}$ isotopes for a direct experimental measure of the isotopic shift in neutron radii. We have also noted in this work that the detailed distribution of neutrons, beyond just the RMS radius, is of some importance. This implies that we may still have to rely on the nuclear models for an extraction of the electroweak parameters. As discussed earlier, the use of alternative electroweak probes, such as parity violating (polarized) electron scattering at intermediate energies, [36] would be of obvious value for independently extracting the desired neutron distribution.

We can turn the problem around, however, and note that an accurate measurement of $\bar{x}$ from high energy experiments presents a unique opportunity to extract the isotopic neutron radius shifts from atomic experiments cleanly, and hence test the nuclear models. The situation is quite analogous to the extraction of changes in charge radii from atomic isotope shifts. 


\section{ACKNOWLEDGMENTS}

We are grateful to C. Chinn, R. Furnstahl, N. Van Giai, M. Girod, I.B. Khriplovich, J. Martorell, M. Musolf, E. Ormand, P.-G. Reinhard, P. Ring, B. Serot, and D. Sprung for valuable discussions and, in some cases, the sharing of calculational data. This work

is supported in part by U. S. Department of Energy grants DOE/ER-06-91ER40561 and DOE/ER-DE-FG06-88ER40427, and by NSF Grant PHY 8922274. 


\section{REFERENCES}

[1] E.N. Fortson, Y. Pang and L. Wilets, Phys. Rev. Lett. 65, 2857 (1990).

[2] S. A. Blundell, W. R. Johnson and J. Sapirstein, Phys. Rev. Lett. 65, 1411 (1990).

[3] V. A. Dzuba, V. V. Flambaum and O. P. Sushkov, Phys. Lett. A141, 147 (1989); A. M. Martensson-Pendrill, J. Phys. (Paris) 46, 1949 (1985); C. Bouchiat and C. A. Piketty, Europhys. Lett. 2, 511 (1986).

[4] V. A. Dzuba, V. V. Flambaum and I. B. Khriplovich, Zeitschrift für Physik D1, 243 (1986); C. Monroe, W. Swann, H. Robinson and C. E. Wieman, Phys.Rev. Lett. 65, 1571 (1990).

[5] M. C. Noecker, B. P. Masterson and C. E. Wieman, Phys. Rev. Lett. 61, 310 (1988); M. A. Bouchiat et al., Phys. Lett. 144B, 463 (1984); E. A. Hinds, in Atomic Physics 11 edited by S. Haroche et al., Proc. of the 11th Int. Conf. on Atomic Physics, Paris, 1988 (World Scientific, Singapore, 1989), p.151.

[6] M.A. Bouchiat and C.C. Bouchiat, J. Phys. [Paris] 35, 899 (1974); 36, 493 (1975); Phys. Lett. B48, 111 (1974).

[7] See e.g. E.N. Fortson and L. Wilets, Adv. in Atomic and Molec. Phys. 16, 319 (1980); E.N. Fortson and L.L. Lewis, Phys. Rep. 113, 289 (1984); M.A. Bouchiat, A. Pottier Science 234, 1203 (1986); E.D. Commins, Phys. Scr. 36, 468 (1987); P. Sandars, Phys. Scr. 36, 904 (1987).

[8] EMC Collaboration, J. Ashman et. al., Phys. Lett. B206, 364 (1988).

[9] J. F. Donnoghue and C.R. Nappi, Phys. Lett. fB206, 364 (1988); J. F. Donnoghue, Ann. Rev. Nucl. Part. Sci. 39, 1 (1989).

[10] D.B. Kaplan and A. Manohar, Nucl. Phys. B310, 527 (1988).

[11] S. Weinberg, Phys. Rev. D5 , 1412 (1972); G. Feinberg, Phys. Rev. D12, 3575 (1975); 
J.D. Walecka, "Weak Interaction Physics - 1977", A.I.P. Conference Proceedings no. 37, American Institute of Physics, New York, 125 (1977).

[12] R.L. Jaffe, Phys. Lett. B229, 275 (1989).

[13] T. T. S. Kuo and G. E. Brown, Nucl. Phys. 85, 40 (1966).

[14] J. Dechargé and D. Gogny, Phys. Rev. C21, 1568 (1980).

[15] P. Quentin and H. Flocard, Ann. Rev. Nucl. and Part. Sci. 28, 523 (1978).

[16] L. Wilets, D. L. Hill and F. W. Ford, Phys. Rev. 91, 1488 (1953).

[17] B.D. Serot and J.D. Walecka, Adv. Nucl. Phys. 16, 1 (1986).

[18] J. Achtzehnter and L. Wilets, Phys. Rev. C38, 5 (1988).

[19] W.D. Meyers and K.H. Schmidt, Nucl. Phys. A410, 61 (1983).

[20] F. Garcias, M. Casas and J. Martorell, Nucl. Phys. A490, 348 (1988); J. Martorell (private communication).

[21] J. Friedrich and P.-G. Reinhard, Phys. Rev. C33, 335 (1986).

[22] M. Girod, C. Chinn, J. Dechargé (private communication).

[23] P.-G. Reinhard (private communication).

[24] P.-G. Reinhard, Rept. Prog. Phys. 52, 439 (1989).

[25] W. Marciano and J.L. Rosner, Phys. Rev. Lett. 65, 2973 (1990).

[26] M. Peskin and T. Takeuchi, Phys. Rev. Lett 65, 964 (1990).

[27] See, e.g., D. London and J.L. Rosner, Phys. Rev. D34, 1530 (1986).

[28] D.C. Kennedy and Paul Langacker, Phys. Rev. Lett. 65, 2967 (1990).

[29] M.M. Sharma, and P. Ring, Phys Rev. C45, 2514 (1992). 
[30] L. Ray and P.E. Hodgson, Phys. Rev. C20, 2403 (1979); G.W. Hoffmann et al., Phys. Rev. C21, 1488 (1980).

[31] C.J. Batty et al., Adv. Nucl. Phys. 19,1 (1989).

[32] L. Ray and G.W. Hoffmann, Phys. Rev. C31, 538 (1985).

[33] N. Hintz, A. Sethi (private communication).

[34] C. Olmer et al., Phys. Rev. C21, 254 (1980); B. Zeidmann, LAMPF Users Group Newsletter Vol 13, No. 1, 91 (1981).

[35] G. R. Satchler, N. Phys. A472, 215 (1987); A. Krasznahorkay et al., Phys. Rev. Lett. 66, 1287 (1991).

[36] T.W. Donnelly, J. Dubach, and I. Sick, Nucl. Phys. A503, 589 (1989).

[37] P. Aufmuth, K. Heilig, and A. Steudel, Atomic Data and Nucl. Data Tables 37, 455 (1987).

[38] C. de Jager, H. de Vries and C. de Vries, Atomic Data and Nucl. Data Tables 36, 495 (1987).

[39] M. Beiner, H. Flocard, N. Van Giai, and P. Quentin, Nucl. Phys. A239, 29 (1975), and N. Van Giai (private communication).

[40] R.C. Thompson et. al., J. Phys. G9, 433 (1983). 


\section{FIGURES}

FIG. 1. Root mean square neutron radius, in fermis, for lead isotopes, plotted versus atomic weight. Models are defined as in Table [1. We also show points for several additional relativistic Hartree parameterizations, NL1, NL06, and NL075. 23,24 Skyrme calculations are connected with dashes to guide the eye. Gogny HFB is connected with dots. Relativistic models are connected with dot-dashes.

FIG. 2. Ratio of neutron to proton radius for lead isotopes, plotted versus atomic weight. Symbols are defined as in Table [ and Fig. 1.

FIG. 3. Neutron correction factor $q_{n}$ for lead isotopes, plotted versus atomic weight. Symbols are defined in Table [I] and Fig. 1. The large error bar on the right side represents the allowed spread corresponding to a $\pm \frac{1}{2} \%$ uncertainty in the weak charge, as given by eqn. (6.8). The vertical position of this error bar is arbitrary.

FIG. 4. Change in $q_{n}$ between ${ }^{202} \mathrm{~Pb}$ and ${ }^{208} \mathrm{~Pb}$, shown versus model weight. (The $\mathrm{x}$ axis is arbitrary) The error bar on the right side represents the allowed spread in the plotted quantity corresponding to a $\pm 1 \%$ uncertainty in $\bar{x}$, as discussed following eqn. (6.11). The vertical position of this error bar is again arbitrary. 


\section{TABLES}

TABLE I. Some properties of ${ }^{202} \mathrm{~Pb}$ relevant to atomic parity violation, for several nuclear models. Properties listed are r.m.s proton radius, neutron radius, ratio $\left(R_{n} / R_{p}\right)$, difference in mean-square charge radius from ${ }^{208} \mathrm{~Pb}, q_{p}$ and $q_{n}$, defined in eqns. (2.9a and b), the normalization factor, $\mathcal{N}$, and a "renormalized norm" defined in (7.1). All distances are in fm. The models listed are Hartree-Fock-Bogolyubov with a Gogny [22] finite-ranged D1S interaction, using a 15 shell spherical harmonic oscillator basis (G:HFB), various parameterizations of the Skyrme interaction [20,21 in the spherical Hartree-Fock approximation, Skyrme A (SkA), star (Sk*), and 3 (Sk3), and relativistic Hartree mean field calculations [23,24] with a nonlinear PL40 parameter set (Rel). The first row contains experimental numbers, where known. [37,38,40] The final row simply indicates the maximum spread among the models.

\begin{tabular}{ccccccccc}
\hline \hline${ }^{202} \mathrm{~Pb}$ & $\sqrt{r_{p}^{2}}$ & $\sqrt{r_{n}^{2}}$ & $R_{n} / R_{p}$ & $\delta r_{c h}^{2}$ & $q_{p}$ & $q_{n}$ & $\mathcal{N}$ & $\mathcal{N}^{\prime}$ \\
\hline Data & & & & $-.330(4)$ & & & & \\
$\mathrm{G}: \mathrm{HFB}$ & 5.409 & 5.519 & 1.020 & -.274 & .90599 & .90318 & 1.0087 & 1.0041 \\
$\mathrm{SkA}$ & 5.431 & 5.607 & 1.032 & -.297 & .90604 & .90141 & 1.0069 & 1.0039 \\
$\mathrm{Sk} *$ & 5.423 & 5.560 & 1.025 & -.296 & .90608 & .90254 & 1.0076 & 1.0039 \\
$\mathrm{Sk3}$ & 5.488 & 5.590 & 1.019 & -.355 & .90638 & .90382 & 1.0018 & 1.0028 \\
$\mathrm{Rel}$ & 5.484 & 5.742 & 1.047 & -.319 & .90618 & .89949 & 1.0023 & 1.0034 \\
\hline Spread & .08 & .2 & .03 & .08 & .0004 & .004 & 0.007 & .001 \\
\hline \hline
\end{tabular}


TABLE II. Same as Table I, for ${ }^{208} \mathrm{~Pb}$.

\begin{tabular}{ccccccccc}
\hline \hline${ }^{208} \mathrm{~Pb}$ & $\sqrt{r_{p}^{2}}$ & $\sqrt{r_{n}^{2}}$ & $R_{n} / R_{p}$ & $\delta r_{c h}^{2}$ & $q_{p}$ & $q_{n}$ & $\mathcal{N}$ & $\mathcal{N}^{\prime}$ \\
\hline Data & $5.453(2)$ & $5.59(4)$ & 1.03 & 0.0 & $.906(1)$ & & $\equiv 1.0$ & \\
$\mathrm{G}: \mathrm{HFB}$ & 5.435 & 5.569 & 1.025 & 0.00 & .90596 & .90260 & 1.0068 & 1.0018 \\
$\mathrm{SkA}$ & 5.459 & 5.670 & 1.039 & 0.00 & .90599 & .90051 & 1.0049 & 1.0016 \\
$\mathrm{Sk} *$ & 5.451 & 5.620 & 1.031 & 0.00 & .90605 & .90176 & 1.0053 & 1.0016 \\
$\mathrm{Sk3}$ & 5.521 & 5.646 & 1.023 & 0.00 & .90636 & .90334 & 0.9992 & 1.0005 \\
$\mathrm{Rel}$ & 5.513 & 5.822 & 1.056 & 0.00 & .90607 & .89813 & 1.0003 & 1.0012 \\
\hline Spread & .1 & .3 & .03 & & .0004 & .005 & 0.008 & .001 \\
\hline \hline
\end{tabular}

TABLE III. Changes in various nuclear properties between ${ }^{202} \mathrm{~Pb}$ and ${ }^{208} \mathrm{~Pb}$. The models are the same as in Table 1. Properties listed are the change in relative neutron to proton radii, the change in proton and neutron correction factors $q_{n}$ and $q_{p}$, and the relative change in the norm. The final column is for the "renormalized norm" defined in eqn. (7.1). The last row shows the maximum spread among the models.

\begin{tabular}{|c|c|c|c|c|c|}
\hline${ }^{202} \mathrm{~Pb} \rightarrow{ }^{208} \mathrm{~Pb}$ & $\Delta R_{n} / R_{p}$ & $\Delta q_{p}$ & $\Delta q_{n}$ & $\Delta \mathcal{N}$ & $\Delta \mathcal{N}^{\prime}$ \\
\hline G:HFB & .0043 & $3 \cdot 10^{-5}$ & $5.8 \cdot 10^{-4}$ & .0020 & .00227 \\
\hline $\mathrm{SkA}$ & .0064 & $5 \cdot 10^{-5}$ & $9.0 \cdot 10^{-4}$ & .0021 & .00232 \\
\hline $\mathrm{Sk}^{*}$ & .0056 & $3 \cdot 10^{-5}$ & $7.8 \cdot 10^{-4}$ & .0022 & .00225 \\
\hline Sk3 & .0040 & $2 \cdot 10^{-5}$ & $4.8 \cdot 10^{-4}$ & .0026 & .00230 \\
\hline Rel & .0091 & $1 \cdot 10^{-4}$ & $1.4 \cdot 10^{-3}$ & .0021 & .00218 \\
\hline Spread $\sim$ & .0051 & $8 \cdot 10^{-5}$ & $9 \cdot 10^{-4}$ & $6 \cdot 10^{-4}$ & $1 \cdot 10^{-4}$ \\
\hline
\end{tabular}

